\title{
Thermal transport and quench relaxation in nonlinear Luttinger liquids
}

\author{
Stanislav Apostolov, ${ }^{1,2}$ Dong E. Liu, ${ }^{1}$ Zakhar Maizelis, ${ }^{1,2}$ and Alex Levchenko ${ }^{1}$ \\ ${ }^{1}$ Department of Physics and Astronomy, Michigan State University, East Lansing, Michigan 48824, USA \\ ${ }^{2}$ A.Ya. Usikov Institute for Radiophysics and Electronics, \\ National Academy of Sciences of Ukraine, 61085 Kharkov, Ukraine
}

(Dated: July 11, 2013)

\begin{abstract}
One-dimensional electrons with a linearized dispersion relation are equivalent to a collection of harmonic plasmon modes, which represent long wavelength density oscillations. An immediate consequence of this Luttinger model of one-dimensional electron systems is the absence of inelastic scattering processes responsible for the relaxation of nonequilibrium states. In a generic nonlinear Luttinger liquid plasmons may decay and thus acquire a finite lifetime. We show that equilibration of plasmons is hierarchical and has profound implications for the dynamics after a thermal quench. We also develop a thermal transport theory and compute thermal conductance of the nonlinear Luttinger liquid by treating the collision integral of plasmons in a manifestly nonperturbative way.

PACS numbers: 71.10.Pm, 72.10.-d, 72.15.Lh, 73.20.Qt, 73.21.Hb
\end{abstract}

\section{INTRODUCTION}

The kinetics of nearly integrable quantum many-body systems is the subject of ongoing study. One-dimensional (1D) systems are special in this regard since several exact solutions are known,,$\frac{1,2}{2}$ and perhaps using them for generic models where integrability is broken weakly makes a good starting point. Integrability ensures that scattering of the particles of an $N$-body system is exactly equivalent to a sequence of pair-particle collisions, and thus the set of incoming momenta for any scattering event coincides with the set of outgoing momenta. Such non-diffractive scattering does not alter the distribution function and is unable to drive the system towards thermal equilibrium. A quantum Newton's cradle, realized with trapped 1D Bose gas, gives the best example for such long-lived out-of-equilibrium quantum states unaffected by binary collisions. $\frac{3}{2}$

The exactly solvable Tomonaga-Luttinger (TL) model $l^{4,5}$ provided a framework for the study of $1 \mathrm{D}$ electron liquids as realized in quantum wires, nanotubes and edge states, see Ref. 6 for a recent review and comprehensive list of references. This model was extremely successful in predicting peculiar properties of such Luttinger liquids (LL), most notable ones being power-law anomalies in the tunneling density of states,,$\frac{7}{\underline{T}}$ and effect of spincharge separation. $\stackrel{8}{*}$ However, it also possesses some serious deficiencies. Within the Luttinger model excitations have infinite lifetime, which implies the lack of equilibration. Interactions in the perfect Luttinger liquid conductor do not affect two-terminal conductance as compared to its noninteracting value $\mathcal{G}_{0}=2 e^{2} / h$. Due to builtin particle-hole symmetry TL model misses completely effects such as thermopower, photo-voltaic response etc.

The resurgence of interests in $1 \mathrm{D}$ electron liquids is triggered by experimental results which clearly fall outside of the LL paradigm. Energy and spatially-resolved tunneling spectroscopy with quantum wires 9.10 and local thermometry with driven quantum Hall edge states 11,12 provided direct evidence for the electronic thermaliza- tion in 1D systems. Transport measurements in low density wires revealed deviations from perfect conductance quantization $13-17$ and violation of the Wiedemann-Franz (WF) law $\stackrel{18.19}{1}$ These observations attracted much theoretical attention and brought to the agenda the new concept of nonlinear Luttinger liquids .20 In the context of the recent studies the subjects of our interest are (i) the microscopic mechanisms of relaxation in the generalized TL description of $1 \mathrm{D}$ electron liquids, which amounts to keeping anharmonic interactions between plasmons, and (ii) the way such liquids transfer energy.

Study of the thermal transport in LL was pioneered by Kane and Fisher ${ }^{21}$ They concluded that: (i) in pure LL the thermal conductance $\mathcal{K}$ does not depend on interaction and coincides with its noninteracting value $\mathcal{K}_{0}=2 \pi^{2} T / 3 h$, so that the Lorentz number $\mathcal{L}=\mathcal{K} / T \mathcal{G}$ is still $\mathcal{L}_{0}=\pi^{2} / 3 e^{2}$ and the WF law holds. (ii) In the presence of a single impurity strong electron backscattering modifies both $\mathcal{G}$ and $\mathcal{K}$ so that $\mathcal{L} / \mathcal{L}_{0}=3 /\left(2 \kappa+\kappa^{2}\right)$ for the Luttinger interaction parameter $1 / 2<\kappa<1$, while the Lorentz number diverges $\mathcal{L} \propto T^{4-2 / \kappa}$ as $T \rightarrow 0$ for $\kappa<1 / 2$. Large violation of the WF law was also predicted for disordered LL on the lattice when umklapp scattering rate exceeds the impurity scattering. ${ }^{22}$ This problem was also analyzed in Refs. 23 25 within the model of inhomogeneous LL with spatially dependent interaction parameter $\kappa(x) \underline{\underline{26}}$ If $\kappa(x)$ varies smoothly on the scale of Fermi wave length then electrons do not suffer backscattering so that conductance is still $2 e^{2} / h$. However, plasmons representing low energy excitation of the LL do backscatter which strongly renormalizes $\mathcal{K}$. Physically this effect can be traced back to the inhomogeneityinduced lifetime of plasmons. $\stackrel{27}{*}$ Note that anharmonic terms neglected within TL model also lead to interaction between plasmons. The effect of these two scattering mechanisms on the plasmon kinetics is very different. Indeed, in the system that lacks translational invariance momentum can be relaxed by scattering off inhomogeneities but taken alone does not lead to thermal equilibrium. In what follows we calculate thermal 
conductance of the genuine (homogeneous) nonlinear LL accounting for the inelastic scattering of plasmons.

\section{MODEL}

The simplest way to visualize charge and heat transport in repulsively interacting electron system is to consider the motion of $1 \mathrm{D}$ Wigner crystal (WC) $\stackrel{28,29}{\underline{2}}$ through the constriction. Wigner crystal, as realized in carbon nanotubes ${ }^{30}$ and quantum wires ${ }^{31,32}$, represents an extreme case of the LL with interaction parameter $\kappa=$ $\pi \hbar \rho^{2} / m s \ll 1$, where $\rho$ is particle density, $m$ is electron mass and $s$ is sound velocity of plasmons. At zero temperature the rigid shift of WC results in interaction independent conductance $\mathcal{G}_{0}$. It is evident that at finite temperatures thermally activated plasmon waves in WC can not affect electrical transport through perfect LL constriction. In fact such corrections require umklapp scattering which are exponentially suppressed at low temperatures and thus can be safely disregarded. However scattering of plasmons may easily alter energy transfer.

We follow recent work ${ }^{33}$ and model the system of strongly interacting spinless electrons by the Hamiltonian (hereafter $\hbar=1$ )

$$
H=\sum_{l} \frac{p_{l}^{2}}{2 m}+\frac{1}{2} \sum_{l \neq l^{\prime}} V\left(x_{l}-x_{l^{\prime}}\right)
$$

where $p_{l}$ and $x_{l}$ are the momentum and coordinate of the $l$ th particle $(l=1, \ldots, N)$, and $V(x)$ is the interaction potential. In calculations we assume screened Coulomb potential $V(x)=\frac{e^{2}}{\epsilon}\left[\frac{1}{|x|}-\frac{1}{\sqrt{x^{2}+4 d^{2}}}\right]$, where $d$ is the distance to the screening gate and $\epsilon$ is the dielectric constant of the host material of the wire. For this model the WC state exists only in the density range $a_{B} / d^{2} \ll \rho \ll a_{B}^{-1}, \stackrel{29}{\underline{ }}$ where $a_{B}=\epsilon / m e^{2}$ is the effective Bohr radius of the material. In the WC picture electrons form a lattice while deviations $u_{l}=x_{l}-l / \rho$ from the corresponding equilibrium sites describe the low energy excitations in the form of electron density waves. Indeed, assuming that relative change of interparticle distance remains small, $\left|u_{l}-u_{l^{\prime}}\right| \ll\left|l-l^{\prime}\right| / \rho$, expanding Eq. (11) to the second order in $u_{l}$, and passing to the oscillator representation with $p_{l}=-i \sum_{q} \sqrt{\frac{m \omega_{q}}{2 N}}\left(b_{q}-b_{-q}^{\dagger}\right) e^{i q l}$ and $u_{l}=\sum_{q} \sqrt{\frac{1}{2 m N \omega_{q}}}\left(b_{q}+b_{-q}^{\dagger}\right) e^{i q l}$, one finds that quadratic part of Eq. (11) takes the usual LL form $H_{0}=$ $\sum_{q} \omega_{q}\left(b_{q}^{\dagger} b_{q}+1 / 2\right)$, where operators $b_{q}^{\dagger}\left(b_{q}\right)$ conventionally created (annihilate) one boson. The plasmon dispersion is given by $\omega_{q}^{2}=(2 / m) \sum_{l} V_{l}^{(2)}[1-\cos (q l)]$, where $V_{l}^{(n)}=\left.\partial_{x}^{n} V(x)\right|_{x=l / \rho}$, which reduces to $\omega_{q}=s|q|$ in the low energy limit $q \rightarrow 0$. We choose to measure all momenta in units of density so that plasmon velocity $s=\left(e^{2} \rho / \varepsilon\right) \sqrt{2 \rho a_{B} \ln (\rho d)}$ has units of energy.

Anharmonic terms in the expansion of Eq. (11) in powers of $u_{l}$ lead to interaction of plasmons and thus man- ifestly break integrability of the model. Quartic nonlinearity and qubic terms iterated to the second order generate two-plasmon scattering process. Ignoring the possibility of coherence between different particle states, i.e. off diagonal elements of the density matrix, plasmons can be described by the distribution function $\mathcal{N}(q, x, t)$, which obeys Boltzmann kinetic equation (BKE) with the collision integral

$$
\begin{array}{r}
I\left[\mathcal{N}_{1}\right]=-\sum_{q_{2} q_{1}^{\prime} q_{2}^{\prime}} W_{Q Q^{\prime}}\left[\mathcal{N}_{1} \mathcal{N}_{2}\left(1+\mathcal{N}_{1^{\prime}}\right)\left(1+\mathcal{N}_{2^{\prime}}\right)\right. \\
\left.-\mathcal{N}_{1^{\prime}} \mathcal{N}_{2^{\prime}}\left(1+\mathcal{N}_{1}\right)\left(1+\mathcal{N}_{2}\right)\right]
\end{array}
$$

where we used shorthand notation $\mathcal{N}_{i}=\mathcal{N}\left(q_{i}, x, t\right)$. The scattering rate $W_{Q Q^{\prime}}=2 \pi\left|A_{Q Q^{\prime}}\right|^{2} \delta_{Q, Q^{\prime}} \delta\left(\Omega-\Omega^{\prime}\right)$ follows from the Fermi's golden rule with the amplitude: $\left|A_{Q Q^{\prime}}\right|^{2}=\left(\frac{\lambda \rho^{2}}{m N}\right)^{2}\left|q_{1} q_{2} q_{1}^{\prime} q_{2}^{\prime}\right|, \frac{33}{3}$ Conventionally, the deltafunctions in $W_{Q Q^{\prime}}$ account for the momentum and energy conservations, with $Q=q_{1}+q_{2}, \Omega=\omega_{q_{1}}+\omega_{q_{2}}$, and similarly for the primed values in the final state. The dimensionless number $\lambda$ is not universal and depends on the choice of interaction potential $V(x)$. For the special potentials that correspond to exactly solvable models 2 it vanishes identically, while $\lambda=-3 / 4$ for the screened Coulomb case $\stackrel{33}{ }$ In order to arrive to that particular form of $A_{Q Q^{\prime}}$ one necessarily has to account for the nonlinearity of the plasmon dispersion $\omega_{q}$ in order to regularize otherwise diverging scattering rate. Collisions encoded by Eq. (2) kinematically allow a process when two comoving plasmons scatters into two counter-propagating ones. Such scattering results in the momentum and energy transfer between the left and right branches of the spectrum, and leads to thermalization.

\section{THERMAL CONDUCTANCE}

Consider now WC quantum wire of length $\ell$ subject to a thermal bias. We assume that right(left) lead is kept at temperature $T_{r(l)}$ such that $T_{r}-T_{l}=\Delta T$. In order to find thermal conductance in such setting we need to calculate energy current carried by plasmons. The latter requires the knowledge of the nonequilibrium plasmon distribution function along the wire, which should follow from the solution of the BKE in a steady state

$$
s_{q} \partial_{x} \mathcal{N}(q, x)=I[\mathcal{N}(q, x)],
$$

were $s_{q}=\partial_{q} \omega_{q}$. Needless to say that finding solution of the integro-differential equation (3) even within the linear response to $\Delta T$ is a complicated problem. We achieve this goal by adopting an approach recently developed for the same problem in the case of weakly interaction 1D electron gas $\stackrel{34.35}{3}^{-1}$ To this end, it is convenient to parameterize distribution function of plasmons in the wire as follows

$$
\mathcal{N}(q, x)=\frac{\theta(q)}{e^{\left(\omega_{q}-u^{R} q\right) / T^{R}}-1}+\frac{\theta(-q)}{e^{\left(\omega_{q}-u^{L} q\right) / T^{L}}-1} .
$$


Here $\theta( \pm q)$ is the step-function, upper indices $R(L)$ refer to right(left) moving plasmons. Parameters $u^{R(L)}$ have meaning of plasmon drift velocities while $T^{R(L)}$ are their effective temperatures. The ansatz made by Eq. (4) can be understood as follows. The scattering process responsible for the energy exchange between plasmons involves three plasmon momenta $q_{1}, q_{2}, q_{1}^{\prime}$ that belong to the same branch (say, right-moving), while forth one $q_{2^{\prime}}$ has to have opposite sign as dictated by the energy and momentum conservation laws. Furthermore, at low temperatures the characteristic scale of $q_{2}^{\prime} \sim(T / s)^{3}$ is parametrically smaller than that of $\left\{q_{1}, q_{2}, q_{1}^{\prime}\right\} \sim T / s$. This implies that redistribution of energy between co-moving plasmons occurs much more efficiently than energy transfer to counter-propagating branch. With this observation at hand, the partially equilibrated form of the plasmon distribution function (44) can be obtained from a general statistical mechanics argument by maximizing the entropy $S=\sum_{q}[(\mathcal{N}+1) \ln (\mathcal{N}+1)-\mathcal{N} \ln \mathcal{N}]$, under the constraints of approximately conserved quantities. The Lagrange multipliers $u^{R(L)}(x)$ and $T^{R(L)}(x)$ are functions of coordinate along the wire that we need to determine. For this purpose we introduce momentum $j_{P}$ and energy $j_{E}$ currents of plasmons

$$
\left\{\begin{array}{c}
j_{P}^{R(L)}(x) \\
j_{E}^{R(L)}(x)
\end{array}\right\}=\int_{-\infty}^{+\infty} \frac{d q}{2 \pi} \theta( \pm q) s_{q}\left\{\begin{array}{c}
q \\
\omega_{q}
\end{array}\right\}\left[\mathcal{N}(q, x)-n_{q}\right]
$$

where $n_{q}=\left(e^{\omega_{q} / T}-1\right)^{-1}$ is equilibrium Bose distribution function. First, we observe that total currents are protected by conservation laws and thus must obey continuity equations

$$
\partial_{x}\left[j_{P}^{R}(x)+j_{P}^{L}(x)\right]=0, \quad \partial_{x}\left[j_{E}^{R}(x)+j_{E}^{L}(x)\right]=0 .
$$

Second, the corresponding currents of right(left)-moving plasmons are not independently conserved and follow from the kinetic equations

$$
\partial_{x}\left[j_{P}^{R}(x)-j_{P}^{L}(x)\right]=2 \dot{p}^{R}, \quad \partial_{x}\left[j_{E}^{R}(x)-j_{E}^{L}(x)\right]=2 \dot{\varepsilon}^{R}
$$

where quantities $\dot{p}^{R}$ and $\dot{\varepsilon}^{R}$ are respective rates of momentum and energy change, which have to be computed from the collision integral Eq. (2). Within the linear response to applied thermal bias $\Delta T$ we expand $\mathcal{N}(q, x)$ in Eq. (41) to the leading order in $u^{R(L)}(x)$ and $\delta T^{R(L)}(x)=T^{R(L)}(x)-T$, and calculate currents from Eq. (5). When combined with Eqs. (6) and (7) this gives us a system of four coupled linear first order differential equations that govern spatial evolution of the Lagrange multipliers that parameterize $\mathcal{N}(q, x)$ in Eq. (4). Specifically we find

$$
\begin{aligned}
& g_{1} \partial_{x} \vartheta_{+}+g_{2} \partial_{x} \eta_{-}=0, \quad \partial_{x} \vartheta_{-}+g_{1} \partial_{x} \eta_{+}=0 \\
& g_{1} \partial_{x} \vartheta_{-}+g_{2} \partial_{x} \eta_{+}=-\frac{\eta_{-}}{\ell_{E}}, \quad \partial_{x} \vartheta_{+}+g_{1} \partial_{x} \eta_{-}=-\frac{\vartheta_{-}}{\ell_{E}} .(9)
\end{aligned}
$$

Here first two equations correspond to the conservation laws in Eq. (6), while the last two correspond to the kinetic equations in Eq. (7). We introduced notations $\vartheta_{ \pm}=\left(\delta T^{R} \pm \delta T^{L}\right) / T$ and $\eta_{ \pm}=\left(u^{R} \pm u^{L}\right) / s$, and two functions $g_{1}=1+\frac{\xi \tau^{2}}{5}+\frac{\xi^{2} \tau^{4}}{2}$ and $g_{2}=1+\frac{2 \xi \tau^{2}}{5}+\frac{8 \xi^{2} \tau^{4}}{7}$, where $\tau=2 \pi T / s$ and $\xi=\frac{(\rho d)^{2} \ln \tau}{2 \ln (\rho d)}$. Keeping the nonlinear terms in the plasmon dispersion $\omega_{q}$ and carrying low-temperature expansion $\tau \ll 1$ is justified in the limit of strong interaction provided that $\kappa \ll(\xi \tau)^{2}$. The relaxation length $\ell_{E}$ in Eqs.(8-9) is given by

$$
\ell_{E}^{-1}=\frac{6}{\pi N T^{3}} \sum_{\substack{q_{1}>0 q_{2}>0 \\ q_{1}^{\prime}>0 q_{2}^{\prime}<0}} \omega_{q_{2}^{\prime}}^{2} W_{Q Q^{\prime}} n_{q_{1}} n_{q_{2}}\left(1+n_{q_{1}^{\prime}}\right)\left(1+n_{q_{2}^{\prime}}\right)
$$

To solve Eqs. (8-9) we need to supplement them by the proper boundary conditions. To find the latter we notice that once electron enters the interacting part of the wire from the leads it breaks up into plasmons. Given its excess energy, determined by the thermal bias $\Delta T$ in our case, conservation laws uniquely determine energy partitioning between right- and left-moving created plasmons. $\stackrel{25,36}{ }$ We thus find

$$
\begin{aligned}
& u^{R}(0)=-\mathcal{R} u^{L}(0), \quad u^{L}(\ell)=-\mathcal{R} u^{R}(\ell), \\
& \delta T^{R}(0)=\mathcal{T} \Delta T / 2+\mathcal{R} \delta T^{L}(0), \\
& \delta T^{L}(\ell)=-\mathcal{T} \Delta T / 2+\mathcal{R} \delta T^{R}(\ell) .
\end{aligned}
$$

The transmission $\mathcal{T}$ and reflection $\mathcal{R}$ coefficients of plasmons from the boundaries separating interacting and noninteracting parts of a wire are given by the Fresnel law: $\mathcal{T}=4 \kappa /(1+\kappa)^{2}$ and $\mathcal{R}=(1-\kappa)^{2} /(1+\kappa)^{2}$. Notice that $\delta T^{R(L)}$ is not simply $\pm \Delta T / 2$, which is essentially due to the quantum analog of the Kapitza boundary thermal resistance $: \frac{37}{}$ This leads us to the final result for the thermal conductance $\mathcal{K}=j_{E} / \Delta T$ :

$$
\frac{\mathcal{K}}{\mathcal{K}_{0}}=\chi g_{1} \frac{\left(1+\chi g_{1}\right)+\left(1-\chi g_{1}\right) e^{-\ell / \ell_{p l}}}{\left(g_{1}+\chi g_{2}\right)+\left(g_{1}-\chi g_{2}\right) e^{-\ell / \ell_{p l}}},
$$

where $\chi=\mathcal{T} /(1+\mathcal{R})$, and interaction-induced inelastic scattering length of plasmons is given by $\underline{38}$

$$
\ell_{p l}^{-1}=\frac{175 s^{4}}{288 \pi^{4} \xi^{2} T^{4}} \ell_{E}^{-1}=c_{\ell} \lambda^{2} \kappa^{2}(T / s)^{5} .
$$

It is instructive to analyze Eq. (14) in various limiting cases. For short wires, $\ell \ll \ell_{p l}$, the effect of plasmon scattering is weak and interaction-induced correction $\delta \mathcal{K}$ to thermal conductance can be obtained by perturbation theory. It scales linearly with the length of the wire, $\delta \mathcal{K} / \mathcal{K}_{0} \propto-\ell / \ell_{p l}$. In contrast, for long wires $\ell \gg \ell_{p l}$, thermalization of plasmons leads to saturation of conductance $\mathcal{K} / \mathcal{K}_{0}=2 \kappa\left[1-\frac{36 \kappa \xi^{2} \tau^{4}}{175}\right]$, where we approximated $\chi \approx 2 \kappa$ for the WC limit $\kappa \ll 1$. Conversely, neglecting thermalization effects but assuming arbitrary interactions we recover from Eq. (14) $\mathcal{K} / \mathcal{K}_{0}=2 \kappa /\left(1+\kappa^{2}\right)$.

We wish to notice that scattering length in Eq. (15) is directly related to the plasmon lifetime $\tau_{p l}=\ell_{p l} / s$, which is in agreement with the results of the recent study 
Ref. 33. In addition, there is one relevant detail which is worth emphasizing. Scattering process considered so far conserves number of plasmons in the initial and final states. This would imply that plasmons acquire chemical potential, which is of course relaxed by the other inelastic processes. The most relevant one involves two plasmons scattering into three or vise versa, which is governed by the collision in integral

$$
\begin{aligned}
I\left[\mathcal{N}_{1}\right]= & -\sum_{\substack{q_{2} q_{1}^{\prime} q_{2}^{\prime} q_{3}^{\prime}\\
}} \widetilde{W}_{Q Q^{\prime}}\left[\mathcal{N}_{1} \mathcal{N}_{2}\left(1+\mathcal{N}_{1^{\prime}}\right)\left(1+\mathcal{N}_{2^{\prime}}\right)\left(1+\mathcal{N}_{3^{\prime}}\right)\right. \\
& \left.-\mathcal{N}_{1^{\prime}} \mathcal{N}_{2^{\prime}} \mathcal{N}_{3^{\prime}}\left(1+\mathcal{N}_{1}\right)\left(1+\mathcal{N}_{2}\right)\right]
\end{aligned}
$$

with the amplitude $\left|\widetilde{A}_{Q Q^{\prime}}\right|^{2}=\frac{2 \tilde{\lambda}^{2} \rho^{6}}{s(m N)^{3}}\left|q_{1} q_{2} q_{1}^{\prime} q_{2}^{\prime} q_{3}^{\prime}\right|$, where we find $\tilde{\lambda}=55 / 48$ Kinematics of this process is such that all momenta transferred are of the same order $\left\{q, q^{\prime}\right\} \sim T / s$ and are on the same branch. Thus this process is not relevant for the energy exchange and the thermal transport, nevertheless it leads to a finite scattering length $\ell_{p l}^{-1} \simeq\left(\tilde{\lambda}^{2} / \xi\right) \kappa^{3}(T / s)^{5}$. Surprisingly, it has the same temperature dependence as $\ell_{p l}^{-1}$ in Eq. (15), while naively one would expect to have it to the higher order in $T$ since amplitude and collision integral contain extra powers of $q$. Two length scales are parametrically distinct in interaction strength $\tilde{\ell}_{p l}^{-1} / \ell_{p l}^{-1} \sim \kappa \ll 1$.

\section{THERMAL QUENCH RELAXATION}

Up to this point we concentrated on the scattering of plasmon excitations and their contribution to the thermal conductance in generic LL. A related question, which attracted a lot of attention recently, is the effect of interactions on the relaxation of sudden quenches in LL $\underline{40}-\underline{42}$ What is still not completely resolved is the problem of integrability-breaking perturbations on the quench dynamics, decay of currents $\underline{43}-\underline{45}$ and ultimately approach to thermal equilibrium. $\underline{\underline{4}}$ We address these issues in the context of the quenched nonlinear LL by studying its evolution at the time scales exceeding inelastic scattering time.

For the time dependent setting it has been shown in Ref. 33 that in the parametrization $\mathcal{N}(q, t)=n_{q}+$ $g_{q} \phi(q, t)$, where $g_{q}=\sqrt{n_{q}\left(1+n_{q}\right)}$, linearized BKE for the plasmon collision integral Eq. (2) can be reduced to the eigenvalue problem for the following integrodifferential equation

$$
\partial_{t} \phi(p, t)=-\tau_{p l}^{-1} \int_{0}^{\infty} \mathbb{K}\left(p, p^{\prime}\right) \phi\left(p^{\prime}, t\right) d p^{\prime} .
$$

The kernel is given by $\mathbb{K}\left(p, p^{\prime}\right)=\frac{1}{6} p^{2}\left(p^{2}+1\right) \delta(p-$ $\left.p^{\prime}\right)+\frac{p p^{\prime}\left(p+p^{\prime}\right)}{\sinh \left[\pi\left(p+p^{\prime}\right)\right]}-\frac{p p^{\prime}\left(p-p^{\prime}\right)}{\sinh \left[\pi\left(p-p^{\prime}\right)\right]}$ and dimensionless variable $p=s q / 2 \pi T$ parametrizes momentum. Above integral operator has continuous spectrum $\zeta_{\nu}=\nu^{2}\left(\nu^{2}+1\right) / 6$ and can be diagonalized in the basis of the eigenfunctions $\psi_{\nu}(p)=\Delta_{\nu}^{-1}\left[\left(2 \nu^{2}-1\right) \delta(p-\nu)+\frac{3 p}{\sinh [\pi(p+\nu)]}+\frac{3 p}{\sinh [\pi(p-\nu)]}\right]$ where the norm is $\Delta_{\nu}=\sqrt{\left(\nu^{2}+1\right)\left(4 \nu^{2}+1\right)}$, such that $\phi(p, t)=\int_{0}^{\infty} \alpha_{\nu} \psi_{\nu}(p) e^{-\zeta_{\nu} t / \tau_{p l}} d \nu$. Expansion coefficients should be determined from the initial conditions $\alpha_{\nu}=$ $\int_{0}^{\infty} \phi(p, 0) \psi_{\nu}(p) d p$. Imagine now a situation that excess energy is suddenly added to the electron liquid which generates heat current. In the actual experiments this could be realized either by selective tunneling of high energy carriers into the quantum wire or by local Joule heating via low conductance quantum point contacts. Relaxation than, in principle, can be monitored by the local thermometry based on the thermoelectric effect. We thus interested in calculating the decay of the energy current carried by (say, right-moving) plasmons. Since Eq. (17) was derived by linearizing plasmon dispersion and furthermore neglecting momentum transferred to left-movers $q_{2}^{\prime} \rightarrow 0$ the eigenmodes of Eq. (17) possess a spurious property that $j_{E}^{R}$ is conserved. To overcome this issue we calculate energy transfer rate $\dot{\varepsilon}^{R}$ directly from the initial collision integral Eq. (2) in the basis of the approximate eigenmodes $\psi_{\nu}(q)$ by keeping transferred momentum explicitly

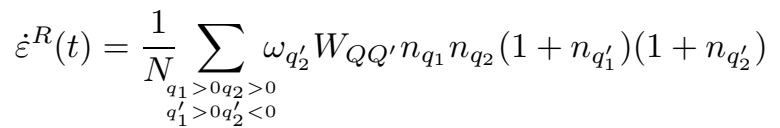

$$
\begin{aligned}
& {\left[\phi\left(q_{1}, t\right) / g_{q_{1}}+\phi\left(q_{2}, t\right) / g_{q_{2}}-\phi\left(q_{1}^{\prime}, t\right) / g_{q_{1}^{\prime}}-\phi\left(q_{2}^{\prime}, t\right) / g_{q_{2}^{\prime}}\right] \text {. }}
\end{aligned}
$$

We choose initial condition assuming that right-moving plasmons are hot $\phi^{R}(q, 0)=g_{q} \omega_{q} \delta T^{R}(0) / T^{2}$. At long times, $t \gg \tau_{p l}$, high-energy plasmons already equilibrated, however thermal plasmons with characteristic momenta $q \sim T / s$ are not yet and their eigenmode expansion at that limit can be approximated by $\phi(p, t) \approx$ $\phi(p, 0) e^{-p^{2} t / 6 \tau_{p l}}-\frac{6 p}{\sinh (\pi p)} \int_{0}^{\infty} \phi(\nu, 0) e^{-\nu^{2} t / 6 \tau_{p l} l} d \nu$, which amounts of taking $\nu \ll 1$ limit of $\psi_{\nu}(p)$. Recalling that $j_{E}^{R} \propto \dot{\varepsilon}^{R}$ one can readily extract long time asymptote of the energy current flux from Eq. (18), which when normalized to the total injected current reads

$$
j_{E}^{R}(t) / j_{E}^{R}(0) \simeq\left(\tau_{p l} / t\right)^{3 / 2},
$$

and exhibits non-exponential decay. The power-exponent in $j_{E}^{R}(t)$ is not universal and depends on the initial condition, however, the power-law dependence at long times is generic feature of the thermal quench relaxation in nonlinear Luttinger liquids.

\section{SUMMARY}

We have presented the study of the thermal transport in the generic $1 \mathrm{D}$ electron system beyond its integrability limit. Combined effects of the energy partitioning and thermalization of plasmons modify thermal conductance of the nonlinear LL in the nontrivial way. The leading order relaxation process stems from the two-plasmon collisions, however complete equilibration is achieved by inelastic scattering that do not conserve number of plasmon modes. Interestingly, both scattering rates have the 
same temperature dependence $\propto T^{5}$. The same physics is responsible for the thermal quench relaxation. We conclude that at the time scales exceeding interactioninduced lifetime of plasmons thermal currents display non-exponential decay, which can be rooted to the fact that eigenvalues of the collision integral for the plasmon scattering cover continuous spectrum of excitations.

\section{Acknowledgment}

We would like to thank Norman Birge and Mark Dykman for reading and commenting on the paper. This work was supported by Michigan State University and in part by ARO through contract W911NF-12-1-0235.
1 D. C. Mattis, The Many-Body Problem: An Encyclopedia of Exactly Solved Models in One Dimension, World Scientific Publishing, 1992).

2 B. Sutherland, Beautiful models: 70 years of exactly solved quantum many-body problems, (World Sci. Pub., 2004).

3 T. Kinoshita, T. Wenger, and D. Weiss, Nature 440, 900 (2006).

${ }^{4}$ S. Tomonaga, Prog. Theor. Phys. 5, 544 (1950).

5 J. M. Luttinger, J. Math. Phys. 4, 1154 (1963).

${ }^{6}$ V. V. Deshpande, M. Bockrath, L. I. Glazman, and A. Yacoby, Nature 464, 209 (2010).

7 L. Venkataraman, Y. S. Hong, and P. Kim, Phys. Rev. Lett. 96, 076601 (2006).

8 O. M. Auslaender, H. Steinberg, A. Yacoby, Y. Tserkovnyak, B. I. Halperin, K. W. Baldwin, L. N. Pfeiffer, and K. W. West, Science 308, 88 (2005).

9 Y.-F. Chen, T. Dirks, G. Al-Zoubi, N. O. Birge, and N. Mason, Phys. Rev. Lett. 102, 036804 (2009).

10 G. Barak, H. Steinberg, L. N. Pheiffer, K. W. West, L. Glazman, F. von Oppen, and A. Yacoby, Nat. Phys. 6, 489 (2010).

11 G. Granger, J. P. Eisenstein, and J. L. Reno, Phys. Rev. Lett. 102, 086803 (2009).

12 C. Altimiras, H. le Sueur, U. Gennser, A. Cavanna, D. Mailly, and F. Pierre, Nat. Phys. 6, 34 (2010).

13 K. J. Thomas, J. T. Nicholls, M. Y. Simmons, M. Pepper, D. R. Mace, and D. A. Ritchie, Phys. Rev. Lett. 77, 135 (1996); K. J. Thomas, J. T. Nicholls, N. J. Appleyard, M. Y. Simmons, M. Pepper, D. R. Mace, W. R. Tribe, and D. A. Ritchie, Phys. Rev. B 58, 4846 (1998).

14 A. Kristensen, H. Bruus, A. E. Hansen, J. B. Jensen, P. E. Lindelof, C. J. Marckmann, J. Nygard, C. B. Sorensen, F. Beuscher, A. Forchel, and M. Michel, Phys. Rev. B 62, 10950 (2000).

15 S. M. Cronenwett, H. J. Lynch, D. GoldhaberGordon, L. P. Kouwenhoven, C. M. Marcus, K. Hirose, N. S. Wingreen, and V. Umansky, Phys. Rev. Lett. 88, 226805 (2002).

16 D. J. Reilly, G. R. Facer, A. S. Dzurak, B. E. Kane, R. G. Clark, P. J. Stiles, R. G. Clark, A. R. Hamilton, J. L. O'Brien, N. E. Lumpkin, L. N. Pfeiffer and K. W. West, Phys. Rev. B 63, 121311(R) (2001).

17 R. Crook, J. Prance, K. J. Thomas, S. J. Chorley, I. Farrer, D. A. Ritchie, M. Pepper, C. G. Smith, Science 312, 1359 (2006).

18 O. Chiatti, J. T. Nicholls, Y. Y. Proskuryakov, N. Lumpkin, I. Farrer, and D. A. Ritchie, Phys. Rev. Lett. 97, 056601 (2006).

19 N. Wakeham, A. F. Bangura, X. Xu, J.-F. Mercure, M. Greenblatt, and N. E. Husseya, Nat. Commun. 2, 396 (2011).
20 A. Imambekov and L. I. Glazman, Science 323, 228 (2009); A. Imambekov, T. L. Schmidt, and L. I. Glazman, Rev. Mod. Phys. 84, 1253 (2012).

21 C. L. Kane and M. P. A. Fisher, Phyes. Rev. Lett. 76, 3192 (1996).

22 A. Garg, D. Rasch, E. Shimshoni, and A. Rosch, Phys. Rev. Lett. 103, 096402 (2009).

23 R. Fazio, F. W. J. Hekking, and D. E. Khmelnitskii, Phys. Rev. Lett. 80, 5611 (1998).

24 I. V. Krive, Low. Temp. Phys. 24, 377 (1998).

25 D. B. Gutman, Y. Gefen, and A. D. Mirlin, Phys. Rev. B 80, 045106 (2009).

26 D. L. Maslov and M. Stone, Phys. Rev. B 52, R5539 (1995).

27 A. Gramada and M. E. Raikh, Phys. Rev. B 55, 7673(1997).

28 H. J. Schulz, Phys. Rev. Lett. 71, 1864 (1993).

29 K. A. Matveev, Phys. Rev. B 70, 245319 (2004).

30 V. V. Deshpande and M. Bockrath, Nat. Phys. 4, 314 (2008).

31 W. K. Hew, K. J. Thomas, M. Pepper, I. Farrer, D. Anderson, G. A. C. Jones, and D. A. Ritchie, Phys. Rev. Lett. 102, 056804 (2009).

32 M. Yamamoto, H. Takagi, M. Stopa, and S. Tarucha, Phys. Rev. B 85, 041308(R) (2012).

33 K. A. Matveev, A. V. Andreev, and M. Pustilnik, Phys. Rev. Lett. 105, 046401 (2010); J. Lin, K. A. Matveev, and M. Pustilnik, Phys. Rev. Lett. 110, 016401 (2013).

34 A. Levchenko, T. Micklitz, Z. Ristivojevic, and K. A. Matveev, Phys. Rev. B 84, 115447 (2011).

35 T. Micklitz and A. Levchenko, Phys. Rev. Lett. 106, 196402 (2011).

36 T. Karzig, G. Refael, L. I. Glazman, and F. von Oppen, Phys. Rev. Lett. 107, 176403 (2011).

37 E. T. Swartz and R. O. Pohl, Rev. Mod. Phys. 61, 605 (1989).

38 The dimensionless numerical coefficient in Eq. (15) is $c_{\ell}=$ $\frac{2100}{\pi^{9}} \iint_{0}^{\infty} \frac{x^{3} y^{3}(x+y)^{3} d x d y}{\sinh (x) \sinh (y) \sinh (x+y)} \approx 7.61$.

39 S. Apostolov and A. Levchenko, unpublished.

40 A. Iucci and M. A. Cazalilla, Phys. Rev. A 80, 063619 (2009).

41 A. Mitra and T. Giamarchi, Phys. Rev. B 85, 075117 (2012).

42 M. S. Foster, T. C. Berkelbach, D. R. Reichman, and E. A. Yuzbashyan, Phys. Rev. B 84, 085146 (2011).

43 A. Rosch and N. Andrei, Phys. Rev. Lett. 85, 1092 (2000).

44 J. Sirker, R. G. Pereira, and I. Affleck, Phys. Rev. B 83, 035115 (2011).

${ }^{45}$ C. Karrasch, R. Ilan, and J. E. Moore, arXiv:1211.2236

46 A. Polkovnikov, K. Sengupta, A. Silva, and M. Vengalattore, Rev. Mod. Phys. 83, 863 (2011). 